\title{
The study of electrohydrodynamic printing by numerical simulation
}

\author{
Xue Yang ${ }^{1}$, Rui Liu ${ }^{1}$, Lu Li ${ }^{2}$, Zhifu Yin ${ }^{1,2}$, \\ *, Kai Chen ${ }^{3}$, Dong Fang Wang ${ }^{1}$
}

\begin{abstract}
EHD (Electrohydrodynamic) printing is a promising technique for alternative fabrication of highresolution micro- and nanostructures without employment of any molds or photo-masks However, the printing precision can be easily influenced by the printing conditions, such as applied voltage, printing distance (the distance between the nozzle tip and the substrate), and flow rate. Unfortunately, up to now there was no work which analyzed those influencing factors in-depth and systematically by theory and numerical simulation. In this paper, the theory of EHD printing was presented and the effect of applied voltage, printing distance, and flow rate on the width of printed line was analyzed by numerical simulation. The simulation results showed that the width of printed lines is proportional to printing distance, nozzle size, and flow rate. However, it is inversely proportional to the applied voltage.
\end{abstract}

K e y w ords: electrohydrodynamic printing, numerical simulation, electrohydrodynamic theory, printing nozzle

EHD (Electrohydrodynamic) printing is a promising technique for alternative fabrication of highresolution micro- and nanostructures without employment of any molds or photo-masks However, the printing precision can be easily influenced by the printing conditions, such as applied voltage, printing distance (the distance between the nozzle tip and the substrate), and flow rate. Unfortunately, up to now there was no work which analyzed those influencing factors in-depth and systematically by theory and numerical simulation. In this paper, the theory of EHD printing was presented and the effect of applied voltage, printing distance, and flow rate on the width of printed line was analyzed by numerical simulation. The simulation results showed that the width of printed lines is proportional to printing distance, nozzle size, and flow rate. However, it is inversely proportional to the applied voltage.

\section{Introduction}

Direct writing techniques show their great potential in for fabrication of micro and nano-structures with low cost, simplified procedures, low-pollution [1]. Among the direct writing methods (Ink jet printing, fused deposition modeling, stereo lithography apparatus, etc.), EHD (Electrohydrodynamic) printing can fabricate nano-scale patterns only by simple equipments and large size nozzles. The jet/droplet is pulled out by an external electric field rather than pressure. During EHD jet printing, a high voltage is applied on the conductive nozzle. Under such high electric field, the charges in the ink move to the nozzle tip and then aggregate quickly, forming a meniscus at the nozzle tip. When the electric field force is larger than the surface tension of the meniscus, a EHD jet is dragged out from the meniscus [2]. The diameter of the jet or droplet is significantly smaller than the nozzle size/the inner diameter of the nozzle (1/100 to $1 / 10$ times) [3]. EHD printing has been demonstrated to write $2 \mathrm{D}$ even $3 \mathrm{D}$ micro- or nano-scale structures in many applications, such as quantum dots [4], electrodes [5], and scaffolds [6].

It has been demonstrated the factors (nozzle size, applied voltage, printing distance, and flow rate) are the main factors which can influence the jet modes, ie . dripping mode at low flow rate, pulsed jet mode at high printing distance, and multi-jet mode at extremely high voltage[7]. It also has been proved the physical properties of ink, such as viscosity, density, and permittivity, can influence the stability and resolution of EHD printing [8]. By adjusting the physical properties of the ink and the process parameters, EHD printing with resolution down to $1 \mathrm{~m}$ can be performed stably.

To explain the phenomenon and jet modes during EHD printing, many researchers around the world developed their EHD printing theories. Choi employed six dimensionless parameters and characterized the correlation of the dimensionless voltage and the charge relaxation length, the jet diameter of cone-jet mode[9]. Lpez-Herrera proposed a volume-of-fluid method adapting to the solution of the governing equations for two-phase EHD problems[10]. The proposed method allows the analysis of the transient stages occurring during the relaxation of charge from the bulk to the interface [10]. Rogers presented a voltage modulation in EHD printing[11]. Based on this modulation, they proposed a short-time high-

1 School of Mechanical and Aerospace Engineering, Jilin University, Changchun 130012, China, 2 Key Laboratory of Auxiliary Chemistry and Technology for Chemical Industry, Ministry of Education, Shaanxi University of Science and Technology, Xian, 710021, China, 3 School of Mechanical Engineering, Liaoning Technical University, Fuxin, 123000, China, yinzf@jlu.edu.cn 
voltage pulse superimposed over a lower baseline constant voltage, so Taylor cone can be formed and maintained at the tip of the micro capillary nozzle[11]. Collins analyzed the formation of jet flow by numerical simulation, and unequivocally show that electrospray droplets are coulombically stable at the instant and there exists a universal scaling law for droplet charge [12]. Kim investigated the characteristics of spray evolution and deposition patterns because of multiple EHD spraying using numerical simulations. Their study demonstrated the feasibility of a multiplexed EHD spraying system. Above studies play a vital role in controlling EHD printing process and explaining the phenomenon during this process. However, the main influencing factors including applied voltage, printing distance, nozzle size (the inner diameter of the nozzle), and flow rate were not simultaneously analyzed. EHD printing process under different conditions were also not numerical analyzed in-depth and systematically.

In the present work, the EHD printing theory based on Navier-Stokes and electric field equations was presented. The influence of applied voltage, printing distance, nozzle size, and flow rate on the width of printed lines were investigated by numerical simulation.

\section{Theory}

\subsection{Theoretical model EHD printing}

Any kind of fluid during flowing process can be governed and expressed by continuity and momentum conservation equation, which is called Navier-Stokes (N-S) equation. Under rectangular coordinate system, for uncompressible fluid $\left(\frac{\partial \rho}{\partial t}=0\right), \mathrm{N}-\mathrm{S}$ equation reads

$$
\begin{gathered}
\rho \frac{\partial \vec{u}}{\partial t}+\rho \vec{u} \cdot \vec{\nabla} \vec{u}=-\vec{\nabla} p+\vec{f}+\mu \nabla^{2} \vec{u}, \\
\nabla \cdot \vec{u}=0,
\end{gathered}
$$

where, $\rho$ is the density of the fluid, $\vec{u}$ is flow rate of the fluid, $\vec{f}$ is the mass force of the fluid, $p$ is the pressure applied on the fluid, and $\mu$ is the viscosity of the fluid.

For EHD printing, the fluid is actuated by electrical field. Therefore, electrodynamic force should add into (1). According to Collins's inference[12], the fluid under electrical field is

$$
\rho \frac{\partial \vec{u}}{\partial t}+\rho \vec{u} \cdot \nabla \vec{u}=-\vec{\nabla} p+\vec{\nabla} \cdot\left(\overrightarrow{\overrightarrow{\sigma^{f}}}+\overrightarrow{\overrightarrow{\sigma^{e}}}\right)+\vec{f}_{b},
$$

where, $\overrightarrow{\sigma^{f}}$ is viscous stress tensor, $\overrightarrow{\overrightarrow{\sigma^{e}}}$ is the electrostatic Maxwell stress tensor, and $\overrightarrow{f_{b}}$ is the body force of the fluid. Viscous stress tensor can be expressed as

$$
\overrightarrow{\sigma^{f}}=\mu\left[\vec{\nabla} \vec{u}+(\vec{\nabla} \vec{u})^{\top}\right]-\frac{2}{3} \mu(\vec{\nabla} \cdot \vec{u}) \overrightarrow{\overrightarrow{\mathrm{I}}},
$$

where, $\overrightarrow{\vec{I}}$ is the identity tensor. Maxwell stress tensor is then

$$
\overrightarrow{\overrightarrow{\sigma^{e}}}=\varepsilon \vec{E} \times \vec{E}-\frac{\varepsilon}{2} E^{2}\left(1-\frac{\rho}{\varepsilon} \frac{\partial \varepsilon}{\partial \rho}\right) \overrightarrow{\overrightarrow{\mathrm{I}}},
$$

where, $\varepsilon$ is the permittivity, $E$ is magnitude of the electric field. Body force is usually equal to the gravity of the fluid and the electric force applied on the fluid, so body force can be calculated as

$$
\overrightarrow{f_{b}}=\rho \vec{g}+q \vec{E}-\frac{1}{2} E^{2} \vec{\nabla} \varepsilon+\frac{1}{2} \vec{\nabla}\left[\rho\left(\frac{\partial \varepsilon}{\partial \rho}\right) E^{2}\right],
$$

where, $g$ is gravitational acceleration, $q$ is the quantity of electric charge. The above equation is the basic theory which can be used for numerical simulation for EHD printing. According to this equation, electric field distribution, the flow rate of the fluid, and the pressure in the fluid can be calculated.

\subsection{The relationship between printing resolution and printing conditions}

The relationship between printing resolution (the width of printed line) and printing conditions can be estimated by scaling law. According to Chois theory [14], the width of printed line $(w)$ can be predicted by an empirical equation

$$
w \approx \sqrt{\frac{\gamma}{\varepsilon_{0}}} \frac{\sqrt{d_{N}}}{E},
$$

where, $\gamma$ is surface tension of the fluid-air, and $d_{N}$ is the inner diameter of the nozzle. According to Chois theory [14], $E$ can be calculated using a theory of infinite planar counter electrode

$$
E=\frac{4 V}{d_{N} \ln \left(\frac{8 h}{d_{N}}\right)},
$$

where, $V$ is the voltage applied on the nozzle, and $h$ is the printing distance Therefore, in conjunction with (7) and (8), the width of printed line can be estimated by

$$
w \approx 8.4017 \times 10^{4} \frac{\gamma^{1 / 2} d_{N}^{3 / 2} \ln \left(\frac{8 h}{d_{N}}\right)}{V} .
$$

During EHD printing, a jet/droplet is pulled out by an external electric field. Before that time, a conical meniscus is generated at the apex of the nozzle. The formation of the conical meniscus is the precondition to produce a jet/droplet. The magnitude of the electric field at this time is called critical magnitude of the electric field $\left(E_{c}\right)$, while the applied voltage for conical meniscus formation is called critical voltage $\left(V_{c}\right)$. Kebarle indicated that conical meniscus can be formed when the electrostatic force is equal to the surface tension of the ink, So Ec can be approximately calculated as, [15]

$$
E_{c}=\sqrt{\frac{2 \gamma \cos \theta}{\varepsilon_{0} d_{N}}}
$$

where, $\theta$ is the half angle of the Taylor cone, which is $49.3^{\circ}$, and the value of $\varepsilon_{0}$ is $8.854187817 \times 10^{-12}$. In conjunction with (8) and (10), critical voltage can be estimated as

$$
V_{c}=9.5952 \times 10^{4} \sqrt{d_{N} \gamma} \ln \left(\frac{8 h}{d_{N}}\right) .
$$




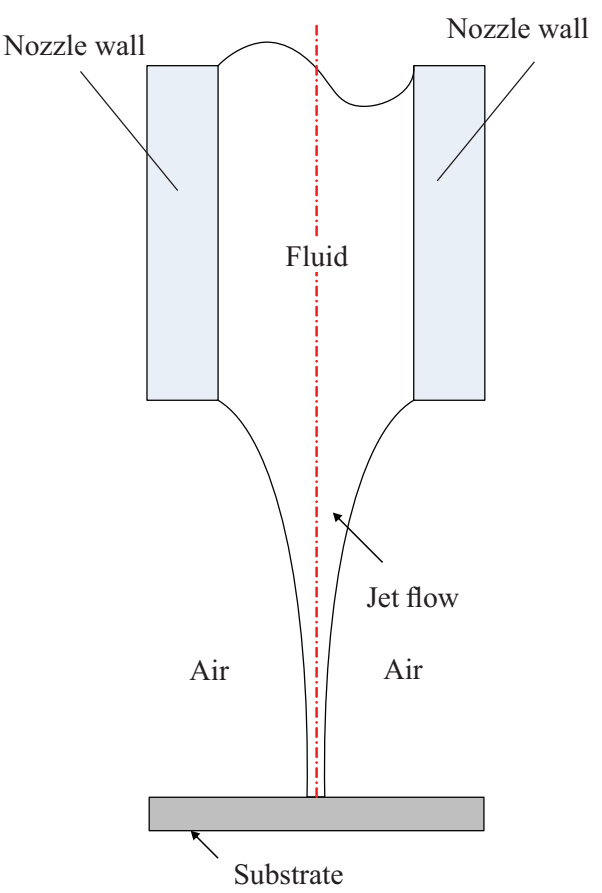

Fig. 1. The geometric model for numerical simulation

\subsection{Numerical simulation for EHD printing process}

The geometry that modeled in this simulation has been showed in Fig. 1. An axisymmetric geometry was modeled in the the finite element domain. The fluid flow and the surface tension were described in terms of volume of fraction (VOF). The nozzle is made by stainless steel, so for simplification the voltage is just applied on the nozzle wall rather than on the fluid. The ground voltage (0 $\mathrm{V})$ is applied on the substrate. Before applied high voltage on the nozzle wall, the area between nozzle and substrate is filled by the air. In this study, glue is used as the printing material. The glue is ultraviolet-cured with the following properties, density of $1098.25 \mathrm{~kg} \mathrm{~m}^{-3}$, viscosity of 330 mpas, surface tension coefficient (glue-air) of 0.036 $\mathrm{Nm}^{-1}$, conductivity of $2.1 \times 10^{-5} \mathrm{sm}^{-1}$, and permittivity of 25.7. During numerical simulation, applied voltage, printing distance, nozzle size, and flow rate are changed, while the printing material is fixed with constant parameters.
The applied voltage is ranging from 900 to $1400 \mathrm{~V}$. The printing distance is ranging from 0.25 to $0.4 \mathrm{~mm}$. The flow rate is ranging from 0.05 to $0.3 \mathrm{ml} / \mathrm{h}$.

\section{Results and discussion}

For EHD printing, there are four main printing modes, which are divided according to the applied voltage. Without or with low voltage, the fluid drips from the nozzle, which is called droplet printing. As the voltage increase, the electrostatic force overcomes the surface tension of the fluid, a fine jet with large diameter can be generated from the nozzle. We call this mode fine jet printing. As applied voltage continuously increases, the diameter of the jet decreases significantly and a cone with $98.6^{\circ}$ can be formed at the apex of the nozzle. Under such voltage, the conejet printing mode was used. It is demonstrated that the half angle of the cone is $49.3^{\circ}[16]$. The jet in this Taylor cone mode become much more stable than that in other modes. Our study in this work is focused on this Taylor cone mode. If the applied voltage continuously increases, there are at least two jets formed at the apex of the needle. The printing turns into multi-jet mode. Needless lines with unanticipated width are printed into the substrate at this mode, so one should prevent from multi-jet during EHD printing.

\subsection{The charge distribution of the fluid during EHD printing}

The formation of EHHD jet can be explained by charge distribution phenomenon when high voltage is applied on the conductive nozzle. Fig. 2 shows the charge distribution of the fluid near the nozzle tip. Melcher reported the permittivity of any fluid is not absolutely zero [17]. There are free charges in the fluid, and the free charges can move under electrical field [17]. When the voltage is applied on the nozzle and ground voltage is applied on the substrate, the free charges in the fluid moves to the tip of the nozzle, Fig. 2(a). With the aggregation of charges, electrostatic force increases, and the fluid is pulled by electrostatic force. Thus, a meniscus is formed near the tip of the nozzle, Fig. 2 (b). When the electrostatic force become large enough, the Taylor cone is generated, Fig. 2(c). Then,

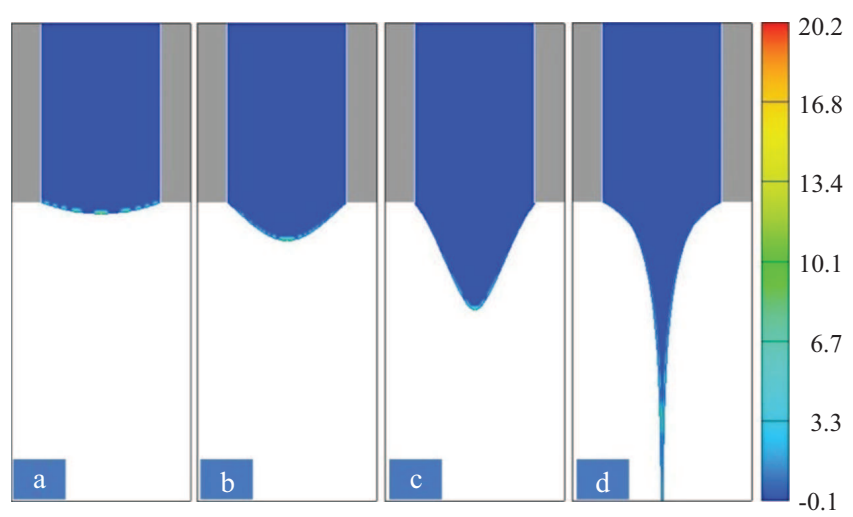

Fig. 2. The charge distribution of the fluid during EHD printing 

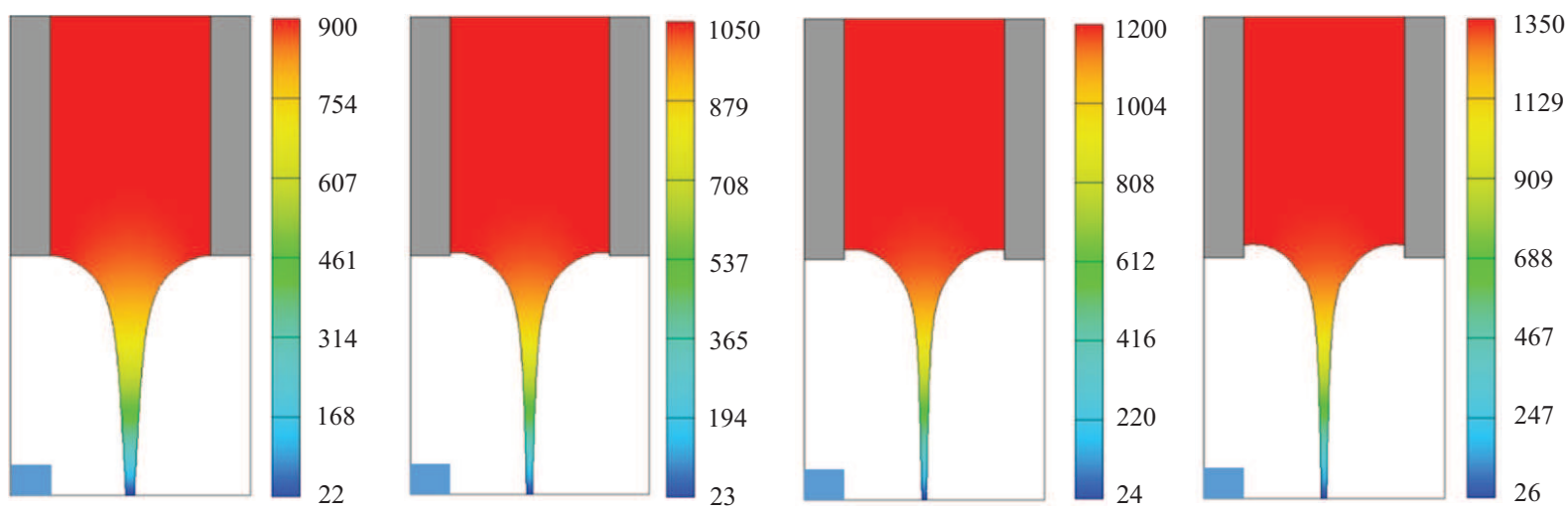

Fig. 3. The simulation of EHD printing under different voltage (a) - $900 \mathrm{~V}$, (b) - $1050 \mathrm{~V}$, (c) $-1200 \mathrm{~V}$, and (d) $-1350 \mathrm{~V}$

a fine jet is pulled out and the fluid is printed onto the substrate, Fig. 2(d), when the the electrostatic force overcomes the surface tension of the fluid.

\subsection{The influence of applied voltage on the width of printed lines}

The simulation was carried out under flow rate of 0.1 $\mathrm{ml} / \mathrm{h}$, nozzle size of $210 \mu \mathrm{m}$, and printing distance of 0.3 $\mathrm{mm}$. The applied voltage was ranging from 900 to 1350 $\mathrm{V}$ with a voltage increment of $150 \mathrm{~V}$. Fig. 3 shows the numerical simulation results. Since the width of printed line usually equal to the diameter of the jet, the width of printed line can be obtained by measuring the diameter of the jet near the substrate. The width of printed lines is $7.2,5.1,3.9$, and $4.2 \mu \mathrm{m}$ for the applied voltage of 900 , $1050,1200,1350 \mathrm{~V}$. It can be seen from the numerical simulation results the width of printed line will decline with the increase of applied voltage. From voltage of 900 to $1200 \mathrm{~V}$, the width decreases obviously. This tendency can be explained by (9) which indicates that the width of printed line is inversely proportional to the applied voltage. However, from voltage of 1200 to $1350 \mathrm{~V}$, the width increases slightly. That can be explained. Under voltage of $1350 \mathrm{~V}$, excessive free charges gather in the jet flow. They repulse to each other due to coulomb force which resulting in the increase of the diameter of the jet. If the applied voltage continuously increases, EHD printing will enter into multi-jet mode because of overlarge coulomb force.

\subsection{The influence of printing distance on the width of printed lines}

Printing distance is defined as the distance between the nozzle tip and the substrate. The simulation was performed with constant flow rate of $0.1 \mathrm{ml} / \mathrm{h}$, nozzle size of $210 \mathrm{~m}$, and applied voltage of $1050 \mathrm{~V}$. The printing distance was ranging from 0.25 to $0.4 \mathrm{~mm}$. Fig. 4 shows the numerical simulation results. The width of printed lines is $4.9,5.1$, and $5.9 \mu \mathrm{m}$ under printing distance of $0.25,0.3$, and $0.4 \mathrm{~mm}$. According to the simulation, from printing distance of 0.25 to $0.4 \mathrm{~mm}$, a larger width of printed line correlates to a higher printing distance. The nozzle size is $210 \mathrm{~m}$, so the value of $\ln \left(8 h / d_{n}\right)$ is larger than zero. This indicates that the width of printed line

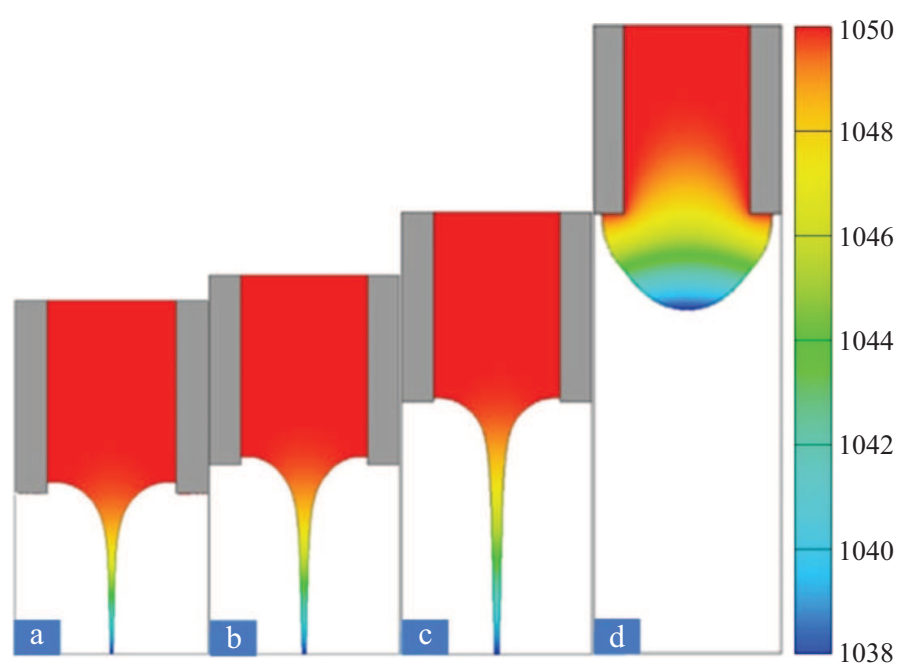

Fig. 4. The simulation of EHD printing under different printing distances: (a) $-0.25 \mathrm{~mm}$, (b) $-0.3 \mathrm{~mm}$, (c) $-0.4 \mathrm{~mm}$, and (d) distance of $0.7 \mathrm{~mm}$ 

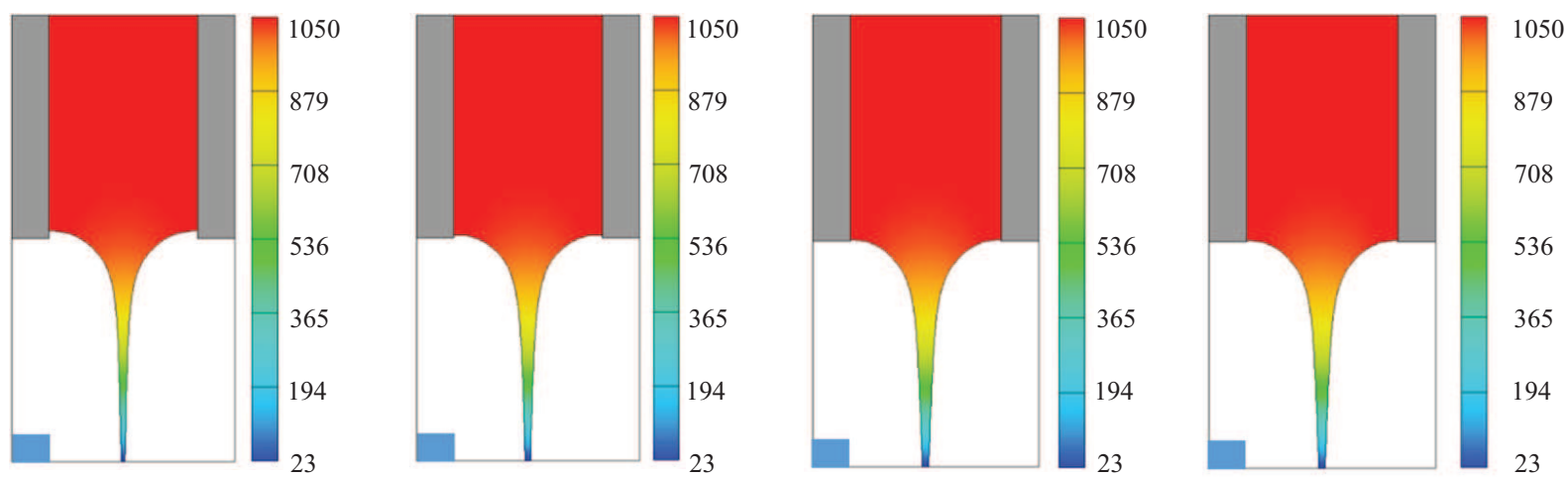

Fig. 5. The simulation of EHD printing under different flow rate: (a) $-0.05 \mathrm{ml} / \mathrm{h},(\mathrm{b})-0.1 \mathrm{ml} / \mathrm{h},(\mathrm{c})-0.2 \mathrm{ml} / \mathrm{h}$, and (d) - flow rate of $0.3 \mathrm{ml} / \mathrm{h}$

is proportional to printing distance. The EHD simulation results and EHD theory are in good agreement with each other. However, the width of printed line cannot infinitely increase. We have discussed the critical magnitude of the electric field $\left(E_{c}\right)$ in section 2.2. If $E<E_{c}$, the EHD jet cannot form. From (8), one can see that $E$ is inversely proportional to $h$. Therefore, with high printing distance there will be no EHD jet on the tip of the nozzle. We have demonstrated this phenomenon in our numerical simulation. Fig. 4 (d) shows that at printing distance of $0.7 \mathrm{~mm}$ the fluid cannot jet from the nozzle at constant flow rate of $0.1 \mathrm{ml} / \mathrm{h}$, nozzle size of $210 \mu \mathrm{m}$, and applied voltage of $1050 \mathrm{~V}$.

\subsection{The influence of flow rate on the width of printed lines}

The influence of flow rate on the width of printed lines was investigated under the following condition: the nozzle size of $210 \mu \mathrm{m}$, applied voltage of $1050 \mathrm{~V}$, and printing distance of $0.3 \mathrm{~mm}$. The flow rate was ranging from 0.05 to $0.3 \mathrm{ml} / \mathrm{h}$. Fig. 5 shows the numerical simulation results. The width of printed lines is $4.7,5.1,6.5,7.4 \mu$ $\mathrm{m}$ under flow rate of $0.05,0.1,0.2$, and $0.3 \mathrm{ml} / \mathrm{h}$. The width of printed lines increases with the improvement of flow rate. According to Ganon-Calvo scaling law the width of printed line can be estimated as [18],

$$
w=\left(\frac{\rho \varepsilon_{0}}{Q^{3}} \gamma K\right)^{1 / 6}
$$

where, $Q$ is flow rate and $K$ is electric conductivity. The above equation was deduced under the assumption of constant other variables, including unchanged fluid properties, constant printing parameters except flow rate. Equation (12) indicates that the width of printed line is proportional to printing distance. This is the same as the numerical simulation results in Fig. 5.

\subsection{The influence of nozzle size on the width of printed lines}

Nozzle size is defined as the inner diameter of the nozzle. The relationship between the nozzle size and the width of printed lines was studied by numerical simulation under the following condition: applied voltage of 1050 $\mathrm{V}$, and printing distance of $0.3 \mathrm{~mm}$, and flow rate of 0.1 $\mathrm{ml} / \mathrm{h}$. The nozzle sizes of 110,120 , and $130 \mathrm{~m}$ were selected. Fig. 6 shows the numerical simulation results. After measurement, the width of printed lines is 3.8, 5.1, 7.6 $\mu \mathrm{m}$ under nozzle size of 110,120 , and $130 \mu \mathrm{m}$. A larger width of printed line correlates to a larger nozzle size. This tendency can also be demonstrated by (9). Here $d_{N}^{3 / 2}$ is an increasing function, while $\ln \left(8 h / d_{N}\right)$ is a decreasing

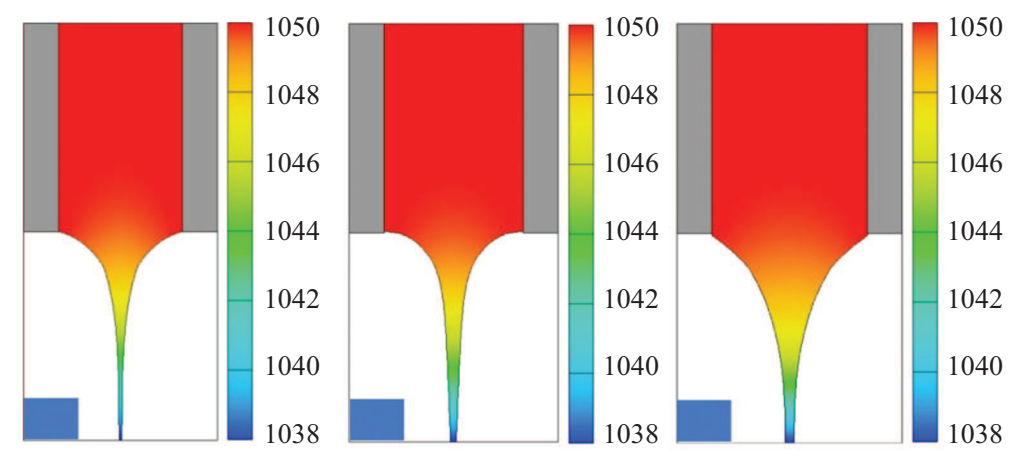

Fig. 6. The simulation of EHD printing under different nozzle sizeamm mlhbmmand cnozzle size of mm 
function. When printing distance $(h)$ is $0.3 \mathrm{~mm}, d_{N}^{3 / 2}$ can increase much more quickly than $\ln \left(8 h / d_{N}\right)$ with the increase of $d_{N}$. Therefore, the printed lines widen when a large size nozzle is used.

\section{Conclusion}

In the present work, the basic theory for EHD printing was presented. The influence of printing distance, flow rate, and nozzle size on the width of printed lines was analyzed by numerical simulation. The results show that the width of printed lines is proportional to printing distance, nozzle size, and flow rate. However, it is inversely proportional to the applied voltage. The relation between the width of printed lines and the printing conditions was explained by derived equations. The EHD simulation results and EHD theory are in good agreement with each other which indicates the feasibility of proposed simulation method.

\section{Acknowledgements}

This project is supported by National Natural Science Foundation of China (No. 51705198, 51775088), Open Fund of Education Ministry Key Laboratory of Auxiliary Chemistry and Technology for Chemical Industry (No. KFKT2019-02), Jilin Province Science and Technology Development Plan (No. 20190103063 JH).

\section{REFERENCES}

[1] T. Qin, J. Y. Dong, and Y. S. Lee, "AC-pulse modulated electrohydrodynamic jet printing and electroless copper deposition for conductive microscale patterning on flexible insulating substrates", Robotics and Computer-Integrated Manufacturing, vol. 43, pp. 179-187, 2017.

[2] J. Park, M. Hardy, S. Kang, K. Barton, K. Adair, D. K. Mukhopadhyay, C. Y. Lee, M. S. Strano, A. G. Alleyne, J. G. Georgiadis, P. M. Ferreira, and J. A. Rogers, "High-resolution electrohydrodynamic jet printing", Nature Materials, vol. 6, no. 10 , pp. 782-789, 2007.

[3] Y. Huang, N. Bu, Y. Duan, Y. Pan, H. Liu, Z. Yin, and Y. Xiong, "Electrohydrodynamic direct-writing", Nanoscale, vol. 5, no. 24, pp. 12007-12017, 2013.

[4] C. Nguyen, T. T. T. Can, and W. S. Choi, "Electrohydrodynamic jet-sprayed quantum dots for solution-processed lightemitting-diodes", Optical Materials Express, vol. 8, no. 12, pp. $3738-3747,2018$

[5] X. Li, G. S. Lee, S. H. Park, H. Kong, T. K. An, and S. H. Kim, "Direct writing of silver nanowire electrodes via dragging mode electrohydrodynamic jet printing for organic thin film transistors", Organic Electronics, vol. 62, pp. 357-365, 2018.

[6] S. Vijayavenkataraman, S. Thaharah, S. Zhang, W. F. Lu, and J. Y. H. Fuh, "Electrohydrodynamic jet 3D-printed PCL/PAA conductive scaffolds with tunable biodegradability as nerve guide conduits (NGCs) for peripheral nerve injury repair", Materials \& Design, vol. 162, pp. 171-184, 2019.

[7] X. Wang, G. Zheng, Z. Luo, and W. Li, "Current characteristics of various ejection modes in electrohydrodynamic printing", Aip Advances, vol. 5, no. 12, pp. 127120, 2015.

[8] L. Guo, Y. Duan, Y. Huang, and Z. Yin, "Experimental Study of the Influence of Ink Properties and Process Parameters on Ejection Volume in Electrohydrodynamic Jet Printing", Micromachines, vol. 9, no. 10, pp. 522, 2018.

[9] A. Lee, H. Jin, H. W. Dang, K. H. Choi, and K. H. Ahn, "Optimization of experimental parameters to determine the jetting regimes in electrohydrodynamic printing", Langmuir, vol. 29, no. 44, pp. 13630-13639, 2013.

[10] M. Lopez-Herrera, S. Popinet, and M. A. Herrada, "A chargeconservative approach for simulating electrohydrodynamic twophase flows using volume-of-fluid", Journal of Computational Physics, vol. 230, no. 5, pp. 1939-1955, 2011.

[11] S. Mishra, K. L. Barton, A. G. Alleyne, P. M. Ferreira, and J. A. Rogers, "High-speed and drop-on-demand printing with a pulsed electrohydrodynamic jet", Journal of Micromechanics and Microengineering, vol. 20, no. 9, pp. 095026, 2010.

12] T. Collins, K. Sambath, M. T. Harris, and O. A. Basaran, "Universal scaling laws for the disintegration of electrified drops", Proceedings of the National Academy of Sciences of the United States of America, vol. 110, no. 13, pp. 4905-4910, 2013.

[13] M. Rahmanpour, R. Ebrahimi, and A. Pourrajabian, "Numerical simulation of two-phase electrohydrodynamic of stable Taylor cone-jet using a volume-of-fluid approach", Journal of the Brazilian Society of Mechanical Sciences and Engineering, vol. 39, no. 11, pp. 4443-4453, 2017.

[14] K. Choi, J. U. Park, O. O. Park, P. M. Ferreira, J. G. Georgiadis, and J. A. Rogers, "Scaling laws for jet pulsations associated with high-resolution electrohydrodynamic printing", Applied Physics Letters, vol. 92, no. 12, pp. 123109, 2008.

[15] P. Kebarle and U. H. Verkerk, "Electrospray: From ions in solution to ions in the gas phase, what we know now", Mass Spectrometry Reviews, vol. 28, no. 6, pp. 898-917, 2009.

[16] J. Zeleny, "Instability of electrified liquid surfaces", Physical review, vol. 10, no. 1, pp. 1, 1917.

[17] R. Melcher and G. I. Taylor, "Electrohydrodynamics: A Review of the Role of Interfacial Shear Stresses", Annual Review of Fluid Mechanics, vol. 1, no. 1, pp. 111-146, 1969.

[18] D. Gao, D. Yao, S. K. Leist, Y. Fei, and J. Zhou, "Mechanisms and modeling of electrohydrodynamic phenomena", ternational Journal of Bioprinting, vol. 5, no. 1, pp. 166, 2019.

Received 12 June 2020 